\title{
Alvéolyse postextractionnelle et membrane résorbable : étude clinique
}

\section{RÉSUMÉ}

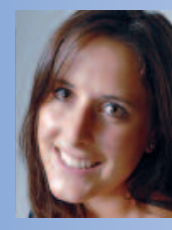

Stéphanie FERNANDEZ

Docteur en chirurgie dentaire,

12 rue Saint-Charles

84000 Avignon.

\section{Paul TRAMINI}

MCU-PH,

UFR d'Odontologie de Montpellier.

Isabelle CALAS-BENNASAR

AHU.,

Département de Parodontologie,

UFR d'Odontologie de Montpellier.

\section{Philippe GIBERT}

PU-PH,

Chef du service d'Odontologie, UFR d'Odontologie de Montpellier,

545 avenue du Pr Jean-Louis Viala,

34295 Montpellier Cedex 5.

Les suites des avulsions ont été largement décrites, entraînant une perte osseuse d'autant plus importante que les corticales sont fines. L'alvéolyse postextractionnelle se produit très rapidement et va se poursuivre pendant plusieurs mois, conduisant à des situations préimplantaires esthétiques et fonctionnelles défavorables. C'est donc avant qu'elle ne se produise qu'il faut intervenir.

La régénération osseuse guidée peut être mise en œuvre au stade des avulsions par l'utilisation de membranes résorbables, permettant la conservation d'un volume osseux de bonne qualité. Les études cliniques contribuent à déterminer l'impact des sites, et des patients sur l'efficacité et la prévisibilité de la régénération osseuse guidée. L'objectif est d'optimiser le processus de sélection des cas et de proposer un choix thérapeutique en termes de développement du protocole de la régénération osseuse guidée.

- régénération osseuse guidée (ROG)

- alvéolyse postextractionnelle

- membranes résorbables 


\section{Introduction}

$>$

L'avulsion dentaire est un acte courant de la pratique quotidienne du chirurgien-dentiste.

Si la conservation des dents, permise par l'apparition de nouvelles techniques, constitue un objectif, l'avulsion dentaire reste le geste thérapeutique ultime concernant l'organe dentaire. Celle-ci doit idéalement s'accompagner en amont d'une réflexion sur le traitement prothétique qu'il convient de réaliser, car cette réflexion peut amener le praticien à adapter sa technique d'extraction.

Les suites des avulsions ont été largement décrites, entraînant une perte osseuse d'autant plus importante que les corticales sont fines.

L'alvéolyse postextractionnelle se produit très rapidement et va se poursuivre pendant plusieurs mois, conduisant à des situations préimplantaires défavorables sur le plan esthétique et fonctionnel. C'est donc avant qu'elle ne se produise qu'il faut intervenir, par des mesures qui peuvent être très simples; des techniques d'avulsion dentaire chirurgicales et atraumatiques, respectant et guidant la cicatrisation osseuse, vont permettre de conserver un volume de crête satisfaisant, apportant des possibilités d'implantation sans recours aux greffes osseuses.

Ces techniques simples, associant traction de lambeaux et sutures hermétiques, doivent être appliquées systématiquement afin de diminuer l'alvéolyse postextractionnelle.

La régénération osseuse guidée peut également être mise en œuvre au stade des avulsions.

Ainsi, I'utilisation de membranes résorbables peut constituer un moyen de conserver ou de reconstruire un volume osseux de bonne qualité.

Le but de cette étude est de comparer le degré de résorption alvéolaire sur le site d'extraction en présence et en l'absence de membrane résorbable.

\section{Matériels et méthodes}

Il s'agit d'une étude clinique comparative entre deux sites d'avulsions similaires, avec et sans membrane résorbable, réalisée au Centre de soins dentaires du CHU de Montpellier.

Les patients présentant deux sites d'avulsion sur le même maxillaire ont été recrutés dans l'étude.

Chaque patient est son propre témoin : un site est laissé tel quel après hémostase, sans suture, et sur l'autre site, après avoir effectué
I'hémostase, une membrane résorbable a été mise en place en vue d'une régénération osseuse. La moitié des patients reçoit une membrane collagénique tandis que l'autre moitié reçoit une membrane Vicryl ${ }^{\circledR}$.

Le site est alors suturé hermétiquement avec couverture de la membrane par le lambeau.

Le site d'avulsion sans ROG (régénération osseuse guidée) n'a pas de visée implantaire. II n'y a pas de perte de chance pour le patient. 


\section{Sélection des patients}

\section{Critères d'inclusion}

Patients ayant fini leur croissance et candidats à une étude après consentement éclairé et assiduité au suivi.

Un projet implantaire est prévu pour le site où la ROG (régénération osseuse guidée) est mise en place.

\section{Matériels}

\section{Les membranes}

> Membrane collagénique : Biomend Extend ${ }^{\circledR}$ (Zimmer Dental) $15 \times 20 \mathrm{~mm}$

La membrane Biomend Extend ${ }^{\circledR}$ est un matériau résorbable indiqué pour la régénération tissulaire guidée. C'est une matrice blanche, compressée et non friable, fabriquée à partir de collagène dérivé du tendon d'Achille de bovin. Le tendon de bovin est connu pour être l'une des sources les plus pures de collagène de type I pouvant être obtenu facilement et produit en quantité commerciale. Sa stérilisation est réalisée par l'oxyde éthylène. C'est une membrane entièrement résorbable éliminant la deuxième phase chirurgicale. La propriété hémostatique du collagène est également à noter.

La membrane va pouvoir être taillée et façonnée dans toutes les formes et dimensions à l'état humide ou sec sans se déchirer, ni se fragmenter. Les pores de la membrane, de 0,004 micron de diamètre, contribuent à retarder la croissance épithéliale durant les premières phases de cicatrisation. Sa nature semi-occlusive va lui permettre d'être perméable aux substances nutri-

\section{Critères de non-inclusion}

Patients avec une maladie parodontale non stabilisée et/ou un contexte de santé générale défavorable et/ou une allergie au triclosan et/ou une hygiène insuffisante et non contrôlée et/ou ayant suivi une antibiothérapie dans les trois derniers mois.

tives essentielles. Enfin, elle va s'incorporer au milieu environnant avant d'être complètement résorbée au bout de 18 semaines.

$$
\begin{aligned}
& \text { > Membrane Vicryl: } \\
& \text { Vicryl }{ }^{\circledR}\left(\text { Polyglactin 910) (Ethicon }{ }^{\circledR}\right) \\
& 30 \times 35 \mathrm{~mm}
\end{aligned}
$$

La membrane Vicryl ${ }^{\circledR}$ est composée d'un copolymère synthétique résorbable de glycolide et de lactide. Ce matériau tissé de façon serrée est formé de fibres non colorées et de composition identique à celle des sutures synthétiques résorbables Vicryl${ }^{\circledR}$ (polyglactine 910). La polyglactine 910 est un copolymère inerte, non antigénique et non pyrogène pouvant jouer son rôle de barrière durant trois à quatre semaines. Sa résorption sera complète au bout de dix semaines environ. II faut noter que les extrémités de la membrane n'étant pas résorbables, elles doivent être éliminées après la fixation de la membrane. Cette membrane comme la collagénique est une barrière temporaire en chirurgie buccale. Elle va limiter la migration des cellules épithéliales en direction apicale et va guider la régénération osseuse. Elle aussi est stérilisée à l'oxyde d'éthylène. 


\section{Le fil de suture}

\section{$>$ Vicryl ${ }^{\circledR}$ Plus antibactérien (4-0)}

Les propriétés recherchées pour des sutures résorbables sont, selon Storch M. et al. (2002), des qualités tactiles favorables (maniabilité), une bonne sécurité des nœuds réalisés et une forte résistance à la traction, ainsi qu'une bonne compatibilité tissulaire (absence d'antigénicité et d'inflammation tissulaire). La nouvelle version de suture en polyglactine 910 enduite de triclosan a été soigneusement évaluée pour vérifier la continuité de ses performances.

Le fil de suture Vicryl ${ }^{\circledR}$ Plus est une suture chirurgicale synthétique, tressée, résorbable et stérile. Il est composé d'acide polyglycolique et d'acide polylactique et est enduit d'un traitement antibactérien : le triclosan. Il protège le fil de suture contre la colonisation bactérienne et prévient ainsi le risque d'infection (Storch M. et al., 2002).

Le triclosan est un antiseptique à large spectre, de la famille des phénols. Il est utilisé depuis plus de trente ans, en toute sécurité dans les produits d'hygiène corporelle. Il faut noter que des essais ont démontré la biocompatibilité du fil enduit de triclosan, c'est-à-dire qu'il n'est pas cytotoxique, qu'il n'entraîne pas d'irritation et qu'il est apyrogène. Par ailleurs, l'addition du triclosan au fil Vicryl ${ }^{\circledR}$ ne modifie pas les caractéristiques physiques, ni les performances de la suture. II n'a été observée aucune différence significative entre Vicryl ${ }^{\circledR}$ et Vicryl ${ }^{\circledR}$ Plus quant à la cicatrisation des plaies (Storch M. et al., 2002). Pourquoi Vicry| ${ }^{\circledR}$ Plus ? Il est bien établi que l'introduction de tout corps étranger dans l'organisme, y compris les sutures, augmente la probabilité d'infection. Les fils de sutures peuvent constituer un vecteur mécanique de migration des bactéries vers la plaie. Malgré le strict respect des règles d'asepsie et les nombreuses mesures d'hygiène entreprises pour diminuer le taux d'infections sur site opératoire, celui-ci reste compris entre moins de $1 \%$ et $10 \%$, selon le type d'intervention chirurgicale (Storch M. et al., 2002).

Les avantages (Storch M. et al., 2002) sont la maniabilité et la souplesse, la sécurité du nœud, le temps de résistance (35 jours), le temps de résorption (70 jours).

\section{Protocole chirurgical}

\section{Avulsion de la dent \\ au niveau du site témoin}

- Anesthésie para-apicale.

- Syndesmotomie.

- Séparation des racines systématique pour les pluriradiculées sauf s'il s'agit de racines fusionnées.

- Élévation.

- Extraction au davier la plus atraumatique possible.
- Curetage et irrigation au sérum physiologique.

- Compression.

\section{Avulsion de la dent}

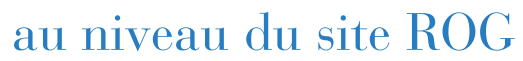

- Même protocole au départ que l'avulsion de la dent au niveau du site témoin.

- Découpe de la membrane pour une meilleure adaptation au site (pour cela réaliser un patron). Le patron dans le cas de la mem- 
brane Biomend Extend ${ }^{\circledR}$ peut être réalisé à l'aide de l'emballage stérile de la membrane en polyéthylène médical haute densité. II est judicieux d'éviter les angles aigus qui pourraient gêner la cicatrisation.

- La membrane doit être hydratée dans une solution saline ou de l'eau stérile. Elle doit dépasser le défaut osseux de 2 à $3 \mathrm{~mm}$ en apical, mésial et distal, pour avoir un appui osseux suffisant gage de stabilité de positionnement de la membrane.

- Fixation de la membrane en lingual par une suture (fig. 1).

- Recouvrement de l'alvéole et application de la membrane en vestibulaire (fig. 2).

- Traction du lambeau vestibulaire et réalisation des sutures. Le lambeau doit recouvrir complètement la membrane. Toutefois pour

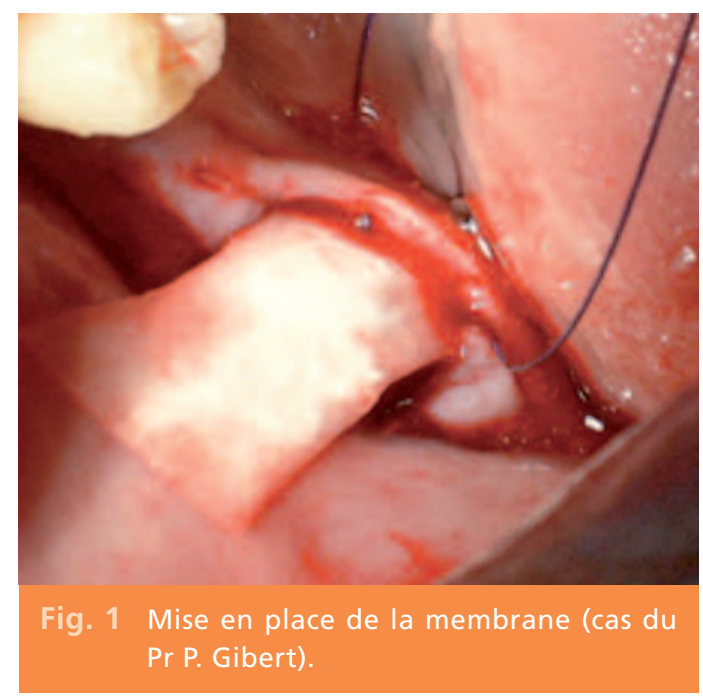

\section{Analyse des résultats}

Les analyses statistiques ont fait appel aux tests de Student appariés et à l'analyse de variance après transformation logarithmique des don- la membrane collagénique une couverture non totale de la membrane ne représente pas une cause d'échec.

\section{Prescriptions}

- Antibiotique : amoxicilline $1 \mathrm{~g}$, un comprimé matin et soir pendant 8 jours.

- Anti-inflammatoire steroïdien : prednisolone, $1 \mathrm{mg} / \mathrm{kg}$ le matin, pendant 3 jours.

- Antalgique : paracétamol $1 \mathrm{~g}$, un comprimé matin, midi et soir pendant 48 heures puis, en cas de douleur, espacer les prises de 6 heures, ne pas dépasser 4 comprimés par jour.

- Bain de bouche : Alodont ${ }^{\circledR}$, ne pas diluer. Un bain de bouche 3 fois par jour après le brossage pendant 8 jours.

- Brosse à dent : chirurgicale $15 / 100$ e pendant 10 jours.

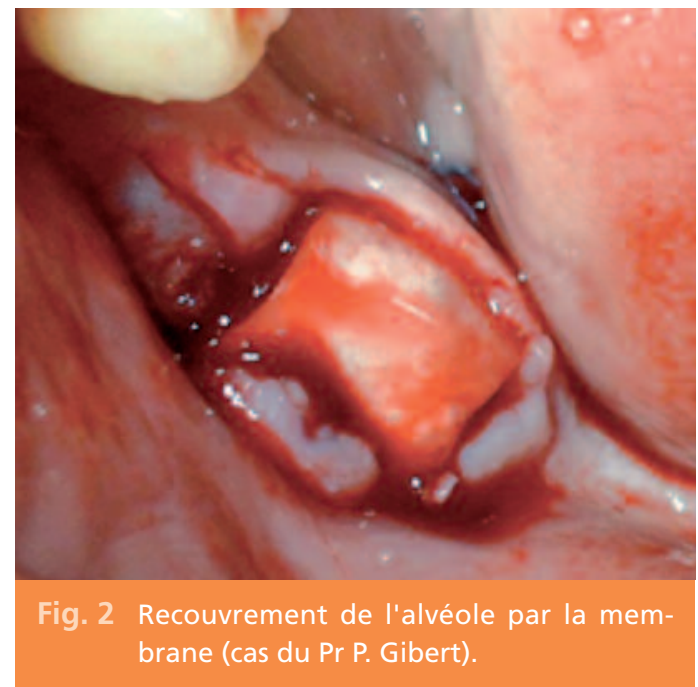

nées. Le seuil de significativité a été fixé à $5 \%$. Tous les tests ont été réalisés au moyen du logiciel de statistique SAS. 


\section{Critère principal d'évaluation}

Il s'agit de la quantification du volume osseux par comparaison de l'os alvéolaire sur cliché dentascan avant avulsion et à quatre mois.

La hauteur et la largeur de l'alvéole ont ainsi été évaluées : la hauteur est définie entre le rebord alvéolaire vestibulaire avant membrane et le bord supérieur du nerf alvéolaire inférieur pour la mandibule, ou le rebord du sinus pour le maxillaire. La largeur est définie entre la corticale externe vestibulaire et palatine ou linguale.

Elles sont ensuite comparées à la hauteur et la largeur de la crête, quatre mois minimum après

\section{Résultats}

\section{Cicatrisation des tissus mous}

En fonction de l'utilisation d'une membrane collagénique ou Vicryl ${ }^{\circledR}$, I'aspect superficiel de la gencive à une semaine paraît meilleur lorsque la membrane utilisée est d'origine collagénique. La dégradation de la membrane Vicryl ${ }^{\circledR}$ par hydrolyse entraîne une inflammation plus importante que la dégradation enzymatique qui fait suite à la mise en place d'une membrane collagénique. Cependant, à 1 mois l'aspect de la gencive concernant la couleur, le volume, la texture, est le même qu'il s'agisse d'une membrane collagénique ou d'une membrane Vicryl ${ }^{\circledR}$.

Le fil Vicry| ${ }^{\circledR}$ Plus, comme le décrivent Storch M. et al. (2002), entraîne initialement une faible réaction inflammatoire se traduisant par une prolifération des cellules du tissu conjonctif. La diminution progressive de la résistance à la traction, ainsi que la résorption du fil se font par la pose de la membrane, toujours par rapport aux repères anatomiques.

C'est par souci de radioprotection que nous avons utilisé pour notre étude la tomographie spiralée (cone beam) réalisant des coupes sagittales et/ou transversales et verticales. Les images radiologiques de notre étude proviennent toutes du même appareil : Scanora Newtom 3G.

\section{Critères secondaires d'évaluation}

C'est la comparaison de la cicatrisation gingivale et muqueuse (état de surface, inflammation, suppuration).

hydrolyse, entraînant une dégradation du copolymère en acides glycolique et acide lactique qui sont assimilés et métabolisés par l'organisme. La résorption entraîne tout d'abord une diminution de la résistance à la traction, puis une disparition du matériau.

\section{Cicatrisation des tissus durs}

Dans cette étude clinique, nous avons suivi 16 patients, 15 ont été inclus dans l'étude, 17 membranes ont été posées, et 34 avulsions ont été réalisées (tableau I).

Les résultats de cette étude montrent une différence significative de la hauteur d'alvéole après cicatrisation entre les sites témoins et les sites tests $(p=0,01)$ (tableau II). De plus, la mise en place d'une membrane collagénique, permet une meilleure préservation, voire une amélioration par rapport à la membrane Vicryl ${ }^{\circledR}$ $(p=0,04)$ (tableau III, fig. 3, et tableau IV). 


\begin{tabular}{|c|c|c|c|c|c|c|}
\hline \multirow{2}{*}{ Cas } & \multirow{2}{*}{ Site témoin / site ROG } & \multicolumn{2}{|c|}{ Site témoin $(\mathrm{H} \times \mathrm{I}) \mathrm{mm}$} & \multicolumn{2}{|c|}{ Site ROG (H x l) mm } & \multirow{11}{*}{ 岂 } \\
\hline & & Avant extraction & Après extraction & Avant extraction & Après extraction & \\
\hline 1 & $48 / 45$ & $10,4 \times 16,3$ & $10,3 \times 14,2$ & $12,6 \times 9,9$ & $17,7 \times 8$ & \\
\hline 2 & $46 / 36$ & $15,8 \times 11$ & $14,5 \times 9,6$ & $12,1 \times 8,1$ & $10,9 \times 9,7$ & \\
\hline 3 & $47 / 36$ & $12,8 \times 9,6$ & $11,9 \times 8,1$ & $11,2 \times 12,5$ & $10,3 \times 9,6$ & \\
\hline 4 & $26 / 16$ & $5,8 \times 12,8$ & $4,8 \times 12$ & $5,5 \times 10$ & $5,5 \times 12$ & \\
\hline 5 & $34 / 14$ & $14,5 \times 6,3$ & $14 \times 5$ & $10,1 \times 7,3$ & $13 \times 6,4$ & \\
\hline 6 & $26 / 16$ & $17,6 \times 8,3$ & $14,8 \times 7,2$ & $18,9 \times 7,8$ & $16,7 \times 7$ & \\
\hline 7 & $46 / 44$ & $12,8 \times 23,4$ & $8,8 \times 19,4$ & $9,4 \times 12,7$ & $10,6 \times 15,3$ & \\
\hline 8 & $45 / 48$ & $11 \times 9$ & $12 \times 10$ & $12 \times 9$ & $14,9 \times 9,2$ & \\
\hline 9 & $45 / 44$ & $11 \times 9$ & $12 \times 10$ & $12 \times 14$ & $13,3 \times 10$ & \\
\hline 10 & $18 / 17$ & $6,1 \times 14,1$ & $6,3 \times 12$ & $6 \times 12,4$ & $6 \times 9,6$ & \multirow{8}{*}{ 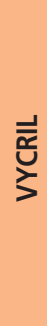 } \\
\hline 11 & $33 / 43$ & $10,3 \times 9$ & $10,8 \times 8,6$ & $15,6 \times 10,5$ & $13 \times 9$ & \\
\hline 12 & $38 / 16$ & $6,3 \times 14$ & $7 \times 14$ & $18,2 \times 12$ & $18,2 \times 10,5$ & \\
\hline 13 & $28 / 37$ & $12,8 \times 11,1$ & $12,8 \times 10,8$ & $13,7 \times 9,8$ & $12,4 \times 9,4$ & \\
\hline 14 & $25 / 23$ & $19,4 \times 10,8$ & $19,1 \times 9,3$ & $5,8 \times 10,7$ & $5,5 \times 10,9$ & \\
\hline 15 & $28 / 16$ & $7 \times 10$ & $5,8 \times 9,5$ & $5,5 \times 12,8$ & $4,8 \times 6,8$ & \\
\hline 16 & $27 / 17$ & $3,5 \times 14,3$ & $4,3 \times 8,9$ & $3,8 \times 14,3$ & $3 \times 13,2$ & \\
\hline 17 & $25 / 15$ & $14,4 \times 9,9$ & $13,3 \times 9,7$ & $12,5 \times 10,5$ & $13,2 \times 11,5$ & \\
\hline
\end{tabular}

\begin{tabular}{|c|c|c|}
\hline \multicolumn{3}{|c|}{$\begin{array}{l}\text { Tableau II Hauteurs moyennes mesurées } \\
\text { sur les différents sites. }\end{array}$} \\
\hline \multicolumn{3}{|c|}{\begin{tabular}{l|l|l} 
Sans membrane & Avec membrane
\end{tabular}} \\
\hline \multicolumn{3}{|c|}{\begin{tabular}{|c|c|c} 
Avant avulsion & 11,5 & 10,9
\end{tabular}} \\
\hline Après avulsion & 10,7 & 11,1 \\
\hline Amélioration & $-0,8$ & $+0,2$ \\
\hline
\end{tabular}

\section{Tableau III Pourcentage des améliora- tions après chirurgie en fonc- tion du type de membrane concernant la préservation de la surface $\mathrm{H} \times \mathrm{I}$.}

\begin{tabular}{c|c|c} 
& Témoins & Tests \\
\hline Collagène & $22,22 \%$ & $66,67 \%$ \\
\hline Vicryl & $25 \%$ & $12,50 \%$ \\
\hline
\end{tabular}

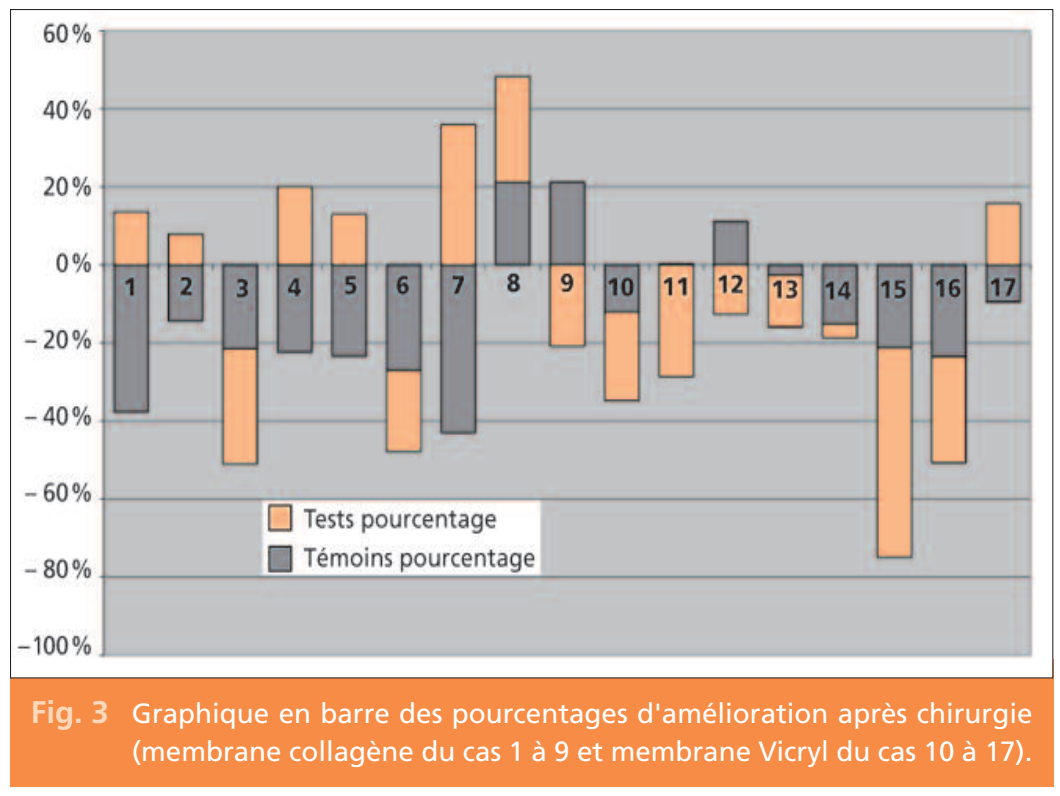

Tableau IV Amélioration ou préservation de la hauteur et de la largeur en fonction du type de membrane. Tableau de Dr S. Fernandez.

\begin{tabular}{c|c|c|c|c} 
& $\begin{array}{c}\text { Amélioration } \\
\text { dans la hauteur } \\
\text { (sites témoins) }\end{array}$ & $\begin{array}{c}\text { Amélioration } \\
\text { dans la largeur } \\
\text { (sites témoins) }\end{array}$ & $\begin{array}{c}\text { Amélioration } \\
\text { dans la hauteur } \\
\text { (sites tests) }\end{array}$ & $\begin{array}{c}\text { Amélioration } \\
\text { dans la largeur } \\
\text { (sites tests) }\end{array}$ \\
\hline Collagène & 2 cas & 2 cas & 6 cas & 4 cas \\
\hline Vicryl & 3 cas & 1 cas & 3 cas & 1 cas \\
\hline
\end{tabular}




\section{Discussion}

Des études (Lekovic V. et al., 1997) ont démontré que la mise en place de membrane collagénique ou polylactide-polyglycolide dans des sites d'extractions permet de réduire la perte osseuse. Ces résultats sont confirmés dans notre étude.

L'analyse de I'alvéole post-extractionnelle va être très importante dans le choix de la technique de préservation de l'alvéole. La classification en trois types, définis par Lekovic V. et al. (1998), en fonction de la présence ou non de tissus mous vestibulaires et de la table externe permettra de choisir la solution de régénération osseuse guidée, suivi éventuellement de greffes de conjonctif, plus complexes.

- Type I: les tissus mous et la table externe sont à leurs niveaux normaux, à hauteur de la jonction amélo-cémentaire des dents adjacentes.

- Type II : les tissus mous restent à hauteur de la jonction amélo-cémentaire des dents adjacentes, mais il y a une perte de hauteur de la table externe.

- Type III : les tissus mous et la table externe sont en position nettement apicale part rapport à la jonction amélo-cémentaire des dents adjacentes.

Les alvéoles de type I représentent une situation idéale pour I'utilisation des membranes de

\section{Conclusion}

Au début des années 1990, un déficit osseux constituait encore une stricte contre indication à l'implantation.

Mais la régénération osseuse guidée permet nettement d'élargir le champ d'application de l'implantologie. régénération osseuse guidée résorbable seule. Les alvéoles de type II qui sont les plus difficiles à diagnostiquer seront les cas les plus justifiables de technique de greffe de comblement alvéolaire de type Bio-Oss ou Pur-Oss maintenue par une membrane résorbable, alors que les alvéoles de type III nécessiteront d'autres techniques que celle de régénération osseuse guidée, le but étant à ce stade de créer de l'os et non de le régénérer ou de le préserver.

Concernant l'esthétique, la fermeture primaire des tissus mous en recourant à la technique du lambeau repositionné coronairement a pour inconvénient de déplacer la ligne muco-gingivale et donc de nuire à l'esthétique.

Dans les cas d'extraction de dents antérieures, où l'esthétique prime, des techniques n'élevant pas de lambeau seront recommandées comme la technique utilisant la membrane Socket Repair ${ }^{\circledR}$. En ne réalisant pas de lambeau, il n’y aura pas de modification de la position de la ligne mucogingivale, ni des contours gingivaux aussi bien vestibulaires qu'interproximaux, ce qui est particulièrement important dans les secteurs esthétiques. Cela permettra d'éviter à nos patients de futures interventions de chirurgies mucogingivale (Roghi C., 2010).

En fonction de la morphologie du défaut osseux et de son étendue, autant dans la hauteur que dans l'épaisseur de la crête résiduelle, nous pourrons choisir le protocole à suivre en tenant compte des limites de chaque technique (Becker et al., 1998) (fig. 4). 
Les études cliniques contribuent à déterminer l'impact des sites, et des patients sur l'efficacité et la prévisibilité de la régénération osseuse guidée. L'objectif est d'optimiser le processus de sélection des cas et de proposer un choix thérapeutique en termes de développement du protocole de la régénération osseuse guidée (Retzpi et Donos, 2010).

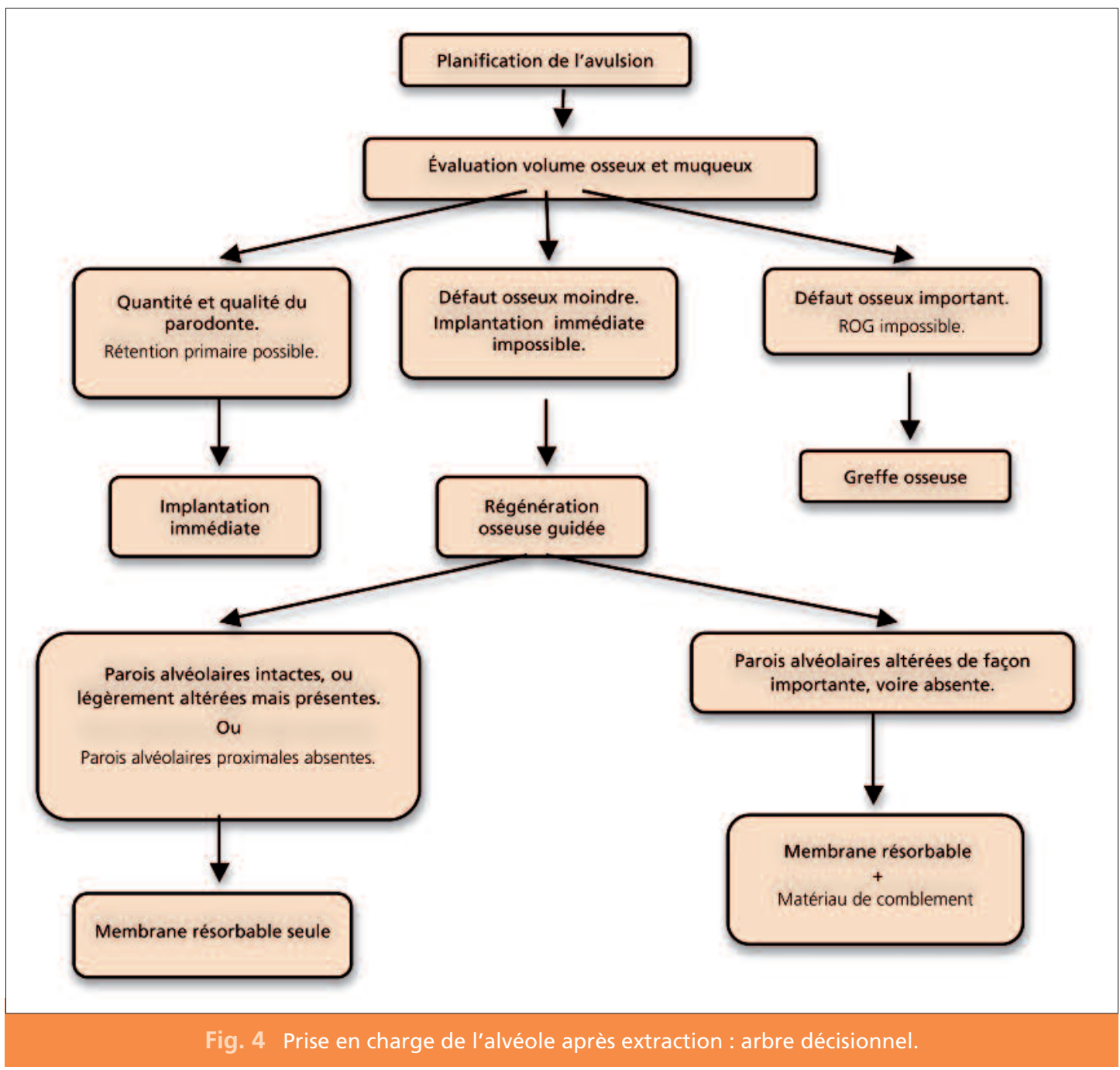

Ouvrages à consulter

- Araújo M, Linder E, Wennström J, Lindhe J. The influence of Bio-Oss Collagen on healing of an extraction socket: an experimental study in the dog. Int J Periodontics Restorative Dent 2008;28(2):123-35.
- Araújo MG, Lindhe J. Dimensional ridge alterations following tooth extraction. An experimental study in the dogs. 
J Clin Periodontol

2005;32(2):212-8.

- Araújo MG, Sukekava F, Wennström JL, Lindhe J. Ridge alterations following implant placement in fresh extraction sockets: an expérimental study in the dog. $\mathrm{J}$ Clin Periodontol 2005;32(6):645-52.

- Becker W, Becker BE. Guided tissue regeneration for implants placed into extraction sockets and for implant déhiscences: surgical techniques and case report. Int J Periodontics Restorative Dent 1990;10(5):376-91.

- Bouquet P, Da Costa-Noble R, Guenand D.

[Periodontology. An Atlas of diagnostic radiology in periodontology (therapeutic impact)]. Chir Dent Fr 1982;52(177): 29-40,45-60,65-7.

- Bousquet P, Gibert P. Gestion parodontale des extractions: pour une conservation des volumes

à visée esthétique et implantaire. Clinic 2009; $\mathrm{N}^{\circ}$ spécial:3-14.

- Bunyaratavej P, Wang HL. Collagen membranes: a review. J. Periodontol 2001;72(2):215-29.

- Buser D, Dula K, Belser U, Hirt HP, Berhold H. Localized ridge augmentation using guided bone regeneration. I. Surgical procedure in the maxilla. Int J Periodontics Restorative Dent 1993;13(1):29-45.

- Buser D, Dula K, Belser U, Hirt HP, Berhold H.

Localized ridge augmentation using guided bone regeneration. II. Surgical procedure in the mandible. Int J Periodontics Restorative Dent 1995;15(1):10-29.
- Buser D, Dula K, Hess D, Hirt HP, Belser UC. Localized ridge augmentation with autografts and barrier membranes. Periodontol 2000 1999;19:151-63.

- Buser D, Dula K, Hirt PH, Schenk RK.

Lateral ridge augmentation using autografts and barrier membranes: a clinical study with $\mathbf{4 0}$ partially edentulous patients. J Oral Maxillofac Surg 1996,54(4):420-32.

- Caneva M, Salata LA, de Souza SS, Bressan E, Botticelli D, Lang NP.

Hard tissue formation adjacent to implants of various size and configuration immediatly placed into extraction sockets: an experimental study in dogs. Clin Oral Implant Res 2010;21(9):885-90.

- Cardaropoli G, Araújo MG, Lindhe J.

Dynamics

of bone tissue formation in tooth extraction sites. An experimental study in dogs. J Clin Periodontol 2003;30(9):809-18.

- Colangelo P, Piattelli A, Barrucci S, Trisi P, Formisano G, Caiazza S.

Bone regeneration guided by résorbable collagen membranes in rabbits: a pilot study. Implant Dent 1993;2(2):101-5.

- Ford HR, Jones P, Gaines B, Reblock K, Simpkins DL. Intraoperative handling and wound healing: controlled clinical trial comparing coated Vicryl ${ }^{\circledR}$ Plus antibacterial suture (coated polyglactin 910 suture with triclosan) with coated Vicryl ${ }^{\circledR}$ suture (coated polyglactin 910 suture). Surg Infect (Larchmt)
2005 Fall;6(3):313-21.

- Gibert P, Bousquet P, Challot E, Boscq L.

Parodontologie : préservation de l'avenir implantaire par régénération osseuse guidée. Chir Dent Fr 2002;1095:54-9.

- Hämmerle CH, Karring T. Guide bone tissue regeneration at oral implant sites. Periodontol 2000 1998;17:151-75.

- Irinakis T.

Rationale for socket preservation after extraction of a single-rooted tooth when planning for future implant placement.

J Can Dent Assoc 2006;72(10):917-22.

- Koong B.

Cone Beam imaging: is this the ultimate imaging modality? Clin Oral Implant Res 2010;21(11):1201-8.

- LaBagnara J.

A review of absorbable suture materials in head and neck surgery and introduction of monocryl: a new absorbable suture.

Ear Nose Throat J 1995;74(6):409-15.

- Le Ray AM.

Le scanner RX :

intérêts et interprétations dans l'étude préimplantaires. Journal de Parodontologie et d'Implantologie Orale 2009;28(1).

- Lekovic V, Camargo PM, Klokkevold PR, et al. Preservation of alveolar bone in extraction sockets using bioabsorbable membranes. J Periodontol 1998;69(9):1044-9.

- Mardas N, Chadha V, Donos N. Alveolar ridge preservation with guided bone regeneration and a synthetic bone sustitute or a bovine-derived xenograft: a randomized, controlled clinical trial. 
Clin Oral Implant Res 2010;21(7):688-98.

- Marinucci L, Lilli C, Baroni T, Becchetti E, Belcastro S, Balducci C, Locci P. In vitro comparison of bioabsorbable and non-resorbable membranes in bone regeneration. J Periodontol 2001;72(6):753-9.

- Melcher AH.

On the repair potential of periodontal tissues. J Periodontol 1976;47(5):256-60.

- Rachlin G, Gardella JP, Boublil M.

Biocompatibility and role of a new resorbable membrane (polyglactin 910-collagen) in periodontal surgery. Preliminary study. J Periodontol 1991;10(3):289-93.

- Retzepi M, Donos N. Guided Bone Regenration: biological principle and therapeutic applications. Clin Oral Implants Res 2010;21(6):567-76.
- Roghi C.

Intérêt de la membrane

Socket Repair ${ }^{\circledR}$

dans la technique

de reconstruction alvéolaire.

Clinic 2010;

NSpécial Septembre:10-14.

- Samson J, et al.

Recommandation

pour la prescription

des anti-inflammatoires

en chirurgie buccale

chez l'adulte.

Méd Buccale Chir Buccale 2008;14(3):129-59.

- Sautier JM, Loty C, Loty S. Biologie de la réparation osseuse : perspective clinique en odontologie.

Inf Dent

1995;38:2955-60.

- Schropp L, Wenzel A, Kostopoulos L, Karring T. Bone healing and soft tissue contour changes following single-tooth extraction: a clinical and radiographic 12-month prospective study.
Int J Periodont Restorative

Dent 2003;23(4):313-23.

- Simion M, Scarano A, Gionso L, Piattelli A.

Guided bone regeneration using resorbable and nonresorbable membranes: a comparative histologic study in humans.

J Oral Maxillofac Implants 1996;11(6):735-42.

- Stimmelmayr M, Reichert TE, Iglhaut G.

Technique de comblement peu invasive intéressant

la zone esthétique.

Titane 2009;6(4):281-9.

- Storch M, Perry LC, Davidson JM, Ward JJ.

A 28-day study of the effect of coated Vicryl ${ }^{\circledR P}$ Plus

Antibacterial Suture (caoted polyglactin 910 suture with triclosan) on wound healing in guinea pig linear incisional skin wounds. Surg Infect (Larchmt) 2002;3 Suppl 1:S89-98.

\section{SUMMARY}

\section{Alveolar bone loss and resorbable membranes: clinic student}

Stéphanie FERNANDEZ, Paul TRAMINI, Isabelle CALAS-BENNASAR, Philippe GIBERT

\section{Keywords \\ - guided bone regeneration \\ - alveolar bone loss \\ - resorbable membranes}

Avulsion's suites have been widely described, resulting in bone loss even more important than the cortex are fine. The post extractionnal bone loss occurs very rapidly and will continue for several months, leading to unfavorable aesthetic and functional situations pre implant. So it is before it happens that we must intervene.

Guided bone regeneration may be implemented at the stage of avulsion by the use of resorbable membranes, allowing the preservation of bone volume of good quality. Clinical studies help to determine the impact sites, and patients about the effectiveness and predictability of guided bone regeneration. The objective is to optimize the selection of cases and to propose a therapeutic choice in terms of development of the protocol guided bone regeneration. 